\title{
Artificial neural network tools for predicting the functional response of ultrafast laser textured/structured surfaces
}

\author{
Luca Baronti $^{1} \cdot$ Aleksandra Michalek $^{2}$ (D) Marco Castellani $^{2} \cdot$ Pavel Penchev $^{2} \cdot$ Tian Long See $^{3} \cdot$ Stefan $_{\text {Dimov }}{ }^{2}$
}

Received: 16 September 2021 / Accepted: 15 December 2021 / Published online: 7 January 2022

(c) The Author(s) 2021

\begin{abstract}
Artificial Neural Networks (ANNs) are well-established knowledge acquisition systems with proven capacity for learning and generalisation. Therefore, ANNs are widely applied to solve engineering problems and are often used in laser-based manufacturing applications. There are different pattern recognition and control problems where ANNs can be effectively applied, and one of them is laser structuring/texturing for surface functionalisation, e.g. in generating Laser-Induced Periodic Surface Structures (LIPSS). They are a particular type of sub-micron structures that are very sensitive to changes in laser processing conditions due to processing disturbances like varying Focal Offset Distance (FOD) and/or Beam Incident Angle (BIA) during the laser processing of 3D surfaces. As a result, the functional response of LIPSS-treated surfaces might be affected, too, and typically needs to be analysed with time-consuming experimental tests. Also, there is a lack of sufficient process monitoring and quality control tools available for LIPSS-treated surfaces that could identify processing patterns and interdependences. These tools are needed to determine whether the LIPSS generation process is in control and consequently whether the surface's functional performance is still retained. In this research, an ANN-based approach is proposed for predicting the functional response of ultrafast laser structured/textured surfaces. It was demonstrated that the processing disturbances affecting the LIPSS treatments can be classified, and then, the surface response, namely wettability, of processed surfaces can be predicted with a very high accuracy using the developed ANN tools for pre- and post-processing of LIPSS topography data, i.e. their areal surface roughness parameters. A Generative Adversarial Network (GAN) was applied as a pre-processing tool to significantly reduce the number of required experimental data. The number of areal surface roughness parameters needed to fully characterise the functional response of a surface was minimised using a combination of feature selection methods. Based on statistical analysis and evolutionary optimisation, these methods narrowed down the initial set of 21 elements to a group of 10 and 6 elements, according to redundancy and relevance criteria, respectively. The validation of ANN tools, using the salient surface parameters, yielded accuracy close to $85 \%$ when applied for identification of processing disturbances, while the wettability was predicted within an r.m.s. error of 11 degrees, equivalent to the static water contact angle (CA) measurement uncertainty.
\end{abstract}

Keywords Artificial neural network $\cdot$ General adversarial network $\cdot$ Feature selection $\cdot$ Laser-induced periodic surface structures $\cdot$ Laser surface texturing

\section{Introduction}

Aleksandra Michalek

AMM752@bham.ac.uk

1 School of Computer Science, University of Birmingham, Edgbaston, Birmingham B15 2TT, UK

2 School of Mechanical Engineering, University of Birmingham, Edgbaston, Birmingham B15 2TT, UK

3 The Manufacturing Technology Centre Ltd, Pilot Way, Ansty Park, Coventry CV7 9JU, UK

Artificial Neural Networks (ANNs) are popular and wellestablished learning systems that employ the principles of biological nervous systems. They are typically composed of several layers of simple nonlinear processing units called neurons. The first layer buffers the input data, after which the signal is processed by a variable number of interconnected hidden layers. Lastly, an output layer provides the ANN's response [1]. Given ANNs' ability to approximate any given function, they are a proven tool with applications 
onto a wide range of industrial problems such as functional prediction or system modelling. Thanks to their learning and generalisation capabilities, ANNs are particularly useful in cases where physical processes are unknown or too complex to be described analytically [2]. ANN development and applications are not limited to specific areas: they can be successfully employed not only in engineering and manufacturing but also in finance, medicine and many other fields [3].

In recent years, ANN developments applicable to laserbased manufacturing processes gained considerable research interest as a novel alternative to physics-based analytical and numerical methods. Most commonly, machine learning algorithms were employed to predict the dimensions of laser-ablated profiles [4-6], along with forecasting surface quality and material removal rates based on the input of the key laser processing parameters [7, 8]. ANNs were also used to identify the optimum laser pulse energy needed to obtain the desired craters' depth and diameter for different materials [9]. Furthermore, ANNs were effectively applied to monitor and control laser processes, and to identify defects by non-destructive detection methods. This was achieved by building a system that identifies defects based on the extracted significant measurement data by employing only image processing [10]. Other methods focused on the analysis of acoustic emissions from the laser-induced plasma [11] or through in situ speckle pattern observations [12]. In all of the various tasks, where the input/output dataset pairs differed significantly, trained neural networks were able to achieve very high prediction accuracy.

The key to obtain good results when applying ANN tools into manufacturing processes is to select an appropriate ANN topology, learning method and suitable data preparation techniques [2]. In addition, a high amount of experimental data is required to train ANNs for optimal performance. Ideally, they should obtain all the relevant information to successfully carry out the desired task. However, building a system from sufficiently big data sets is time-consuming, problematic and in most cases not viable. A common solution to this issue is to augment the available training data, and such approach was already successfully applied in simulating complex systems based only on small experimental datasets [13, 14]. One of the novel augmentation techniques is Generative Adversarial Networks (GANs). They are composed of two convolutional neural networks and were originally designed to generate artificial images that are indistinguishable from the real ones [15]. GANs were already utilised as a predictive visualisation method in laser machining. Laser-ablated topographies were recreated based on spatial laser intensity profiles [16] or by transforming the key laser parameters into predicted 3D surface profiles [17].

Another area where ANNs can be effectively applied is laser structuring/texturing for surface functionalisation.
A particular type of sub-micron structures, generated by ultrafast lasers, are Laser-Induced Periodic Surface Structures (LIPSS). Low Spatial Frequency LIPSS are especially attractive to researchers due to their vast applicability and the wide range of achievable surface functionalities, e.g. modifying wettability, enhancing cell proliferation or structural colouring, to name a few [18]. The functional response of LIPSS surfaces is mostly dictated by their topological characteristics, i.e. periodicity, amplitudes and regularity of ripples. LIPSS are all sensitive to changes in laser processing conditions, in particular in cases where processing disturbances affect the laser structuring process, e.g. when LIPSS are generated on 3D and freeform surfaces. The most common disturbances are variations in the Beam Incident Angle (BIA) and Focal Offset Distance (FOD). The relationship between disturbances and their influence on LIPSS topographies has been studied, and it was shown that BIA affects their periodicity while FOD mostly influences ripples amplitudes $[19,20]$. Thus, any variations in processing conditions due to structuring disturbances during the LIPSS generation affect the surface functionality, too. Typically, the surface responses are analysed experimentally to confirm whether the functional performance is still within acceptable limits [21, 22]. However, obtaining functional performance data from the laser treated surfaces is often time-consuming, limited to specific processing settings, and requires special instruments and measurement setups. Another issue related to LIPSS generation in the presence of processing disturbances is the lack of adequate process monitoring and quality control tools to maintain the process in control. ANNs can offer promising solutions for condition monitoring during the laser structuring process and consequently indirectly to judge whether the surface's functional performance is still within some predefined limits.

In this research, ANN tools were developed for pre- and post-processing of LIPSS topography data, i.e. their areal surface roughness parameters, for two main tasks. The first is the identification of whether any processing disturbances were present during the laser structuring process. The second is the mapping of the LIPSS topographies to their functional responses, here wettability. For both tasks, a small representative experimental dataset augmented with GAN-generated LIPSS topographies was used to develop and train ANN classifiers, while the validation was performed on a larger unseen dataset. The pre-processing step involved the application of feature selection methods to minimise the number of data attributes based on their relevance and redundancy. The next section outlines the experimental methods used to create representative data sets of LIPSS topographies. These data sets are required to develop and validate the proposed ANN tools. Then, the pre-processing methods (GAN and feature selection) and the ANN structure optimisation tools are described, together with the ANN tools developed for 
the two tasks. Subsequently, the experimental results of the implementation of the proposed methods are presented and discussed. Finally, conclusions are made about the effectiveness of the investigated feature extraction methods and ANN tools, and their applicability to the two classification and prediction tasks associated with the use of LIPSS treatments.

\section{Experimental methods}

Laser structuring was performed using an ultrafast Ytterbiumdoped laser source (Satsuma from Amplitude Systems) with a near-infrared wavelength $(\lambda)$ of $1032 \mathrm{~nm}$, pulse duration of $310 \mathrm{fs}$, maximum average power and pulse energy of $5 \mathrm{~W}$ and $10 \mu \mathrm{J}$, respectively. A linearly polarised Gaussian laser beam was focused with a $100 \mathrm{~mm}$ telecentric lens on workpieces to deliver a beam spot size of $40 \mu \mathrm{m}$. The laser processing of surfaces was realised by employing a 3D scan head. A motorised rotational stage was employed, and a dynamic focusing module with a working range of $\pm 3 \mathrm{~mm}$ from the focal plane was used to control the laser focusing for the samples produced with varied BIA. The LIPSS treatments were performed on $1.5 \mathrm{~mm}$ thick, mirror polished, 304 stainless steel plates.

Optimised laser settings and strategy for generating regular and uniform LIPSS obtained from initial trials were used, in particular: peak fluence of $0.28 \mathrm{~J} / \mathrm{cm} 2$, pulse repetition rate of $10 \mathrm{kHz}, 40 \mathrm{~mm} / \mathrm{s}$ scanning speed and $6 \mu \mathrm{m}$ hatching distance between the pulse trains that yielded the pulse distance of $4 \mu \mathrm{m}$ and $6 \mu \mathrm{m}$ in $\mathrm{x}$ and $\mathrm{y}$ direction, respectively. The relatively low scanning speed was chosen due to the limitations of the dynamic focusing module. The schematic representation of the described laser processing strategy is presented in Fig. 1a. The laser processing settings were set constant, while structuring disturbances were present and controlled as shown in Fig. 1b. Square fields of $8 \mathrm{~mm} \times 8 \mathrm{~mm}$ were produced with varying disturbances, i.e. from 0 to $+900 \mu \mathrm{m}$ with an increment of $100 \mu \mathrm{m}$ for FOD, and separately from 0 to $35 \mathrm{deg}$ with an increment of $2.5 \mathrm{deg}$ for BIA. Each field with different set of disturbances was produced three times. Additionally, 15 supplementary LIPSS topographies were produced with the same scanning strategy, without disturbances but with varied peak fluence in the range from near-threshold $0.16 \mathrm{~J} / \mathrm{cm} 2$ to $0.54 \mathrm{~J} / \mathrm{cm} 2$.
Fig. 1 Schematic representation of processing strategy for LIPSS generation with a) optimised processing settings and $\mathbf{b}$ ) when processing disturbances, i.e. FOD and BIA, are present during the laser structuring a)

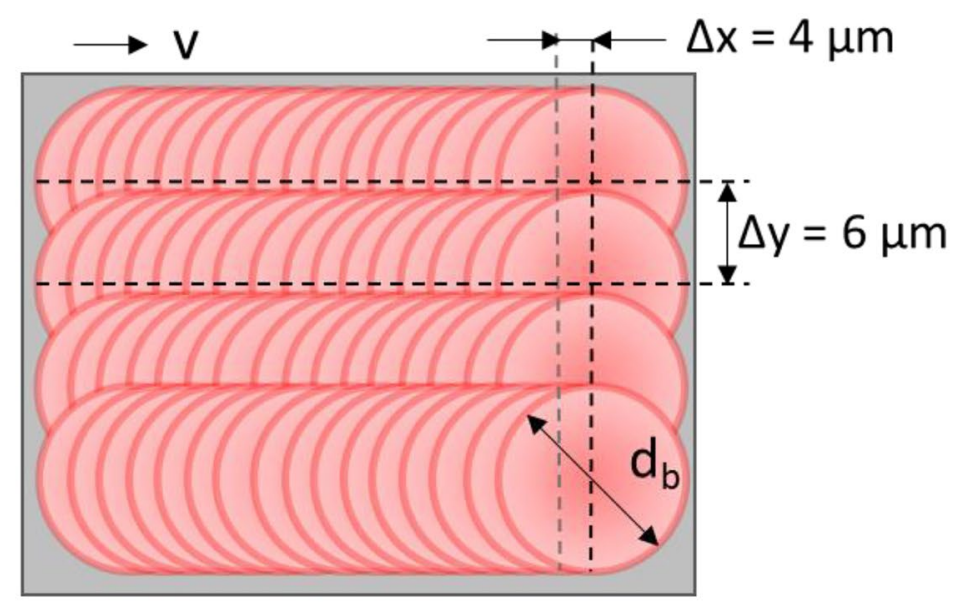

b)

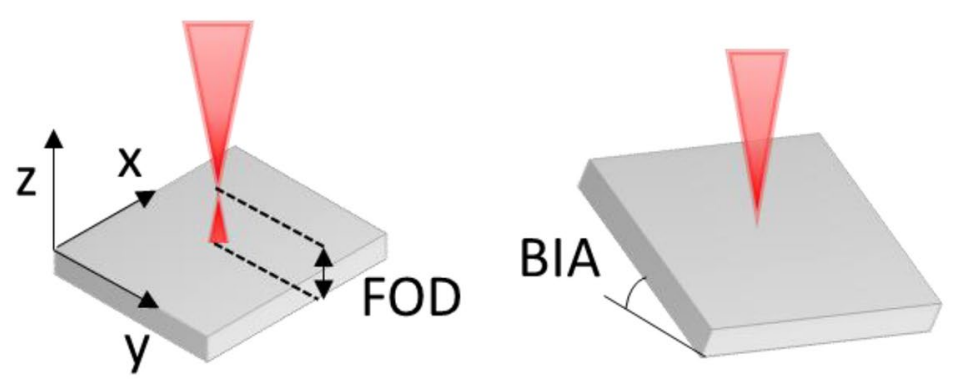

\begin{tabular}{c|c|c} 
Disturbance & FOD & BIA \\
\hline Range, increment & $0-+900 \mu \mathrm{m}$, every $+100 \mu \mathrm{m}$ & $0-35 \mathrm{deg}$, every $2.5 \mathrm{deg}$
\end{tabular}


Table 1 List of areal surface roughness parameters calculated based on LIPSS surface topography data according to ISO 25178 standard and considered as input data for ANN training

\begin{tabular}{lllll}
\hline Types of Parameters & Height & Spatial & Hybrid & Functional \\
\hline Symbol & Sq, Ssk, Sku, Sp, Sv, Sz, S10z, Sa & Sal, Str & Sdq, Sdr & Smr1, Smr2, Sk, Spk, Svk, Vvv, Vvc, Vmp, Vmc \\
\hline
\end{tabular}

The topographies of the LIPSS-treated surfaces were analysed by using an Atomic Force Microscopy (AFM) from Digital Instruments D3100 with NanoScope controller. In total, 87 scans of $20 \mu \mathrm{m} \times 20 \mu \mathrm{m}(256 \mathrm{px} \times 256 \mathrm{px})$ fields were analysed, and all necessary topography data were acquired. Then, each surface sample was used to extract 16 reference images (100 $\mathrm{px} \times 100 \mathrm{px}$ ) by using an overlapping sliding window every $50 \mathrm{px}$. Pre-processed images were fed into the Alicona MeasureSuite software to calculate 21 standardised areal surface roughness parameters according to ISO 25178 . The roughness parameters are the most commonly used to characterise surfaces, i.e. sets of height, spatial, hybrid and functional parameters, and they are listed in Table 1.

The wettability of laser structured surfaces was analysed with the contact angle (CA) goniometer (OCA 15EC, Data Physics $\mathrm{GmbH}$ ). The static CA on each laser-processed surface was measured 4 times employing the sessile drop arrangement for optical measurement of CA by using a drop shape analysis. Droplets of de-ionised water were deposited with $1 \mu \mathrm{l} / \mathrm{s}$ speed to form a droplet of $4 \mu \mathrm{l}$, and then, they were carefully placed on the laser-processed field. Prior to CA measurement, each test sample was carefully cleaned in an ultrasonic bath, first in acetone and then in $99.8 \%$ ethanol solution for $3 \mathrm{~min}$. Next, the analysed surfaces were rinsed with deionised water and dried with compressed air after each bath. The reason for such rigorous sample preparation procedure was the necessity to minimise the effects of varying surface chemistry, and the presence of organic residuals after laser irradiation, which affect the resulting wettability of LIPSS-treated surfaces [23]. All CA tests were repeated more than 6 months after the laser processing while the samples were stored in ambient conditions. The CA of as-received steel substrates was $73.3 \pm 10$ degrees.

The produced samples were split into a small experimental subset, i.e. Set A, and a much larger validation set-Set B. Set A consisted of 18 surface samples, where 5 were produced with varying FOD and another 7 with varying BIA. The remaining 6 samples were chosen from the supplementary set produced with optimised laser settings but varying laser fluence. From each sample, 16 topography images were extracted, for a total of 288 created topographies. Set B comprised the remaining 69 surfaces from the conducted 87 AFM scans. Again, from each scan of the validation set, 16 topography images were created, for a total of 1104 LIPSS topographies.

\section{Artificial neural network tools}

\subsection{General Adversarial Networks for data augmentation}

In this research, GAN, as a novel data augmentation technique, was used to generate additional realistic artificial

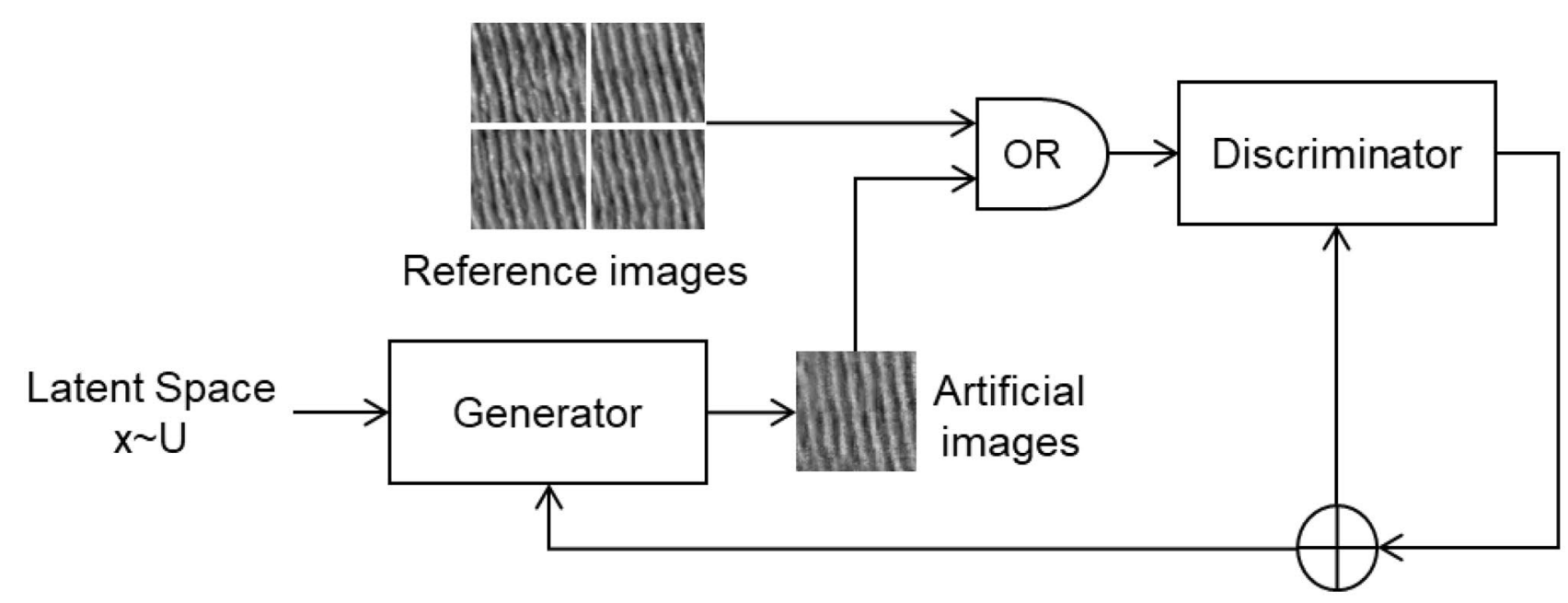

Fig. 2 Schematic representation of GAN used to generate artificial height maps/depth images based on experimental LIPSS topography data. The loss signal of the Generator is the opposite of the loss sig- nal of the Discriminator, which allowed the height maps, created by the Generator, to become progressively more realistic and similar to the real/reference surfaces 
Table 2 Description of the main layers that compose the Generator (with initial size $\mathrm{S}=10$ )

\begin{tabular}{ll}
\hline Layer & Parameters \\
\hline Dense & \# units $=128^{*} \mathrm{~S}^{\wedge} 2$ \\
& activation $=\mathrm{ReLu}$ \\
Reshape & size $=(\mathrm{S}, \mathrm{S}, 128)$ \\
Batch Normalisation & momentum $=0.8$ \\
Upsampling (2D) & factor $=5$ \\
Convolutional & \# filters $=128$ \\
& kernel size $=3$ \\
Batch Normalisation & activation $=$ ReLu \\
Upsampling (2D) & momentum $=0.8$ \\
Convolutional & factor $=2$ \\
& \# filters $=64$ \\
Batch Normalisation & kernel size $=3$ \\
Convolutional & activation $=$ ReLu \\
& momentum $=0.8$ \\
& \# filters $=1$ \\
& kernel size $=3$ \\
& activation $=$ tanh \\
\hline
\end{tabular}

LIPSS topographies based on Set A. The extracted LIPSS topographies were treated as height maps/depth images. The respective AFM data were converted into 16-bit greyscale height maps that contain the coordinates of each point on the surface in a three dimensional Cartesian system, i.e. the known $\mathrm{Z}$ resolution (nm per greyscale value) and pixel size value for $X$ and $Y$ [24].

One GAN was trained separately for each laser structured surface sample, using the 16 extracted height maps as reference images. The schematic representation of a GAN is shown in Fig. 2. The main role of the Generator is to produce artificial images that are indistinguishable from the reference images for the Discriminator, and this is the basis for the training procedure. That is, the Generator's aim is to learn to create images of progressively higher similarity to the reference ones. The aim of the Discriminator is to learn to distinguish the reference images from the artificial ones. After completing the training, the Discriminator was discarded and only the Generator was used to create 20 artificial images. The $100 \mathrm{px} \times 100 \mathrm{px}$ height maps created by the Generator were imported into the Alicona software, and the areal surface roughness parameters were calculated for each of them.

The GAN architecture was determined by trial-and-error during a preliminary process of parameters fine tuning. The detailed learning process is described hereafter. Each artificial image created by the Generator, whose architecture is presented in Table 2, was based on a vector of 100 random scalar values fed as input to the network. Using a sequence of upscaling and convolutional layers, a matrix of $100 \times 100$ elements (normalised in $[-1,1]$ ) was produced. The final image was generated by re-scaling the matrix elements to
Table 3 Description of the main layers that compose the Discriminator

\begin{tabular}{ll}
\hline Layer & Parameters \\
\hline Convolutional & \# filters $=16$ \\
& kernel size $=3$ \\
strides $=2$ & activation $=$ Leaky ReLu (alpha $=0.2)$ \\
& rate $=0.25$ \\
Dropout & \# filters $=32$ \\
Convolutional & kernel size $=3$ \\
& strides $=2$ \\
& activation $=$ Leaky ReLu (alpha $=0.2)$ \\
& rate $=0.25$ \\
Dropout & momentum $=0.8$ \\
Batch Normalisation & \# filters $=128$ \\
Convolutional & kernel size $=3$ \\
& strides $=1$ \\
& activation $=$ Leaky ReLu (alpha =0.2) \\
& rate $=0.25$ \\
Dropout & \# units $=1$ \\
Dense & activation = sigmoid \\
\end{tabular}

16-bits unsigned integers. The Discriminator architecture, described in Table 3, was composed of an alternate stack of convolutional and dropout layers. Both the Generator and Discriminator were trained together using the Adam optimiser, albeit with different learning rates. For each epoch, an equal number of real images (sampled with replacement from the reference images) and artificial images, created by the Generator, were fed to the Discriminator that was trained against a binary label (i.e. real $=1$, fake $=0$ ). The Generator was trained on a complemented value of the Discriminator loss, in a zero-sum fashion. The training parameters are given in Table 4 . To improve the early convergence of the Generator, a measure of noise has been added to the data used by the Discriminator. Each time a reference (i.e. real) image was fed to the Discriminator, the associated label was randomly flipped (with $\mathrm{p}=0.5$ ). This was not performed in the case of the artificial images. This regularisation procedure limited the Discriminator potential of greatly outperforming the Generator in the early stage of the learning

Table 4 Parameters used for the GAN optimisers and learning process

\begin{tabular}{ll}
\hline Parameter & Value \\
\hline epochs & $3 * 10^{\wedge} 3$ \\
batch size & 32 \\
discriminator learning rate & $5^{*} 10^{\wedge}-5$ \\
generator learning rate & $2 * 10^{\wedge}-4$ \\
loss & binary \\
& cross- \\
& entropy \\
\hline
\end{tabular}


process, to the point of hindering its ability to learn to generate good quality images.

Overall, the set of GAN-generated images (henceforth Set GAN) consisted of 360 artificial topographies $(18 \times 20)$; each was described by 21 areal surface roughness parameters and one $\mathrm{CA}$ value. The procedure of assigning the $\mathrm{CA}$ to the GAN topographies (as well as to Set A and Set B) was as follows: the mean $(\mu)$ and standard deviation $(\sigma)$ were calculated for the obtained CA values for each surface sample. Then, one CA value was assigned randomly to each topography from a uniform CA distribution within the interval $(\mu-\sigma, \mu+\sigma)$.

\subsection{Feature selection and ANN structure optimisation}

The feature selection analysis, ANN optimisation and validation procedure were run three times in parallel to assess the usefulness of the GAN-generated artificial LIPSS topographies. By using only the small Set A, it was intended to test the feasibility of performing the whole study using a limited amount of experimental data. Then, the quality of the GAN-generated topographies was assessed based on tests run only on the Set GAN. Finally, the benefits of augmenting the available experimental data with the artificially generated ones were evaluated on the merged Sets A and GAN. All three cases were also validated on Set B.

Feature selection methods were applied to the datasets to filter out redundant and irrelevant attributes among the ISO areal surface parameters and jointly perform ANN structure optimisation [16]. A parameter/feature is considered relevant when it conveys useful information for a given task, and redundant when it does not add additional information that has not been already provided by other parameters. The purpose of feature selection was to find the smallest number of most related areal surface roughness parameters, without significantly reducing the ANN's accuracy for the two specific tasks. The first task was a classification problem, where the ANN had to be trained to detect either the presence of processing disturbances (FOD or BIA in this research), or the use of optimised laser settings during the structuring process (labelled as class $\mathrm{N}$ ). Then, the same group of surface parameters was applied to the second task. The second task amounted to a regression problem, where the ANN had to learn the relationship between the identified group of areal surface roughness parameters, and the static water CA of the laser-treated surfaces. It is important to state that the ability to detect alterations in LIPSS topographies due to any processing disturbances might also help to foresee potential variations in the surface performance. Therefore, the results from the classification task can indicate potential changes in the surface functional response and can be used to trigger some corrective processing routines to keep it within predefined limits.

\subsubsection{Feature redundancy analysis}

Data feature (attribute) redundancy was assessed by using the well-known Pearson correlation coefficient [25]. In this study, two data attributes (areal surface parameters) were considered highly correlated and hence redundant if their correlation coefficient $\left|\rho_{x y}\right|$ was higher than 0.8. After the analysis, redundant parameters were removed sequentially, starting with the one that showed significant similarities with the largest number of other parameters. Once this surface parameter had been removed, the one amongst the remaining that had the largest number of significant similarities with the others was eliminated, and so forth until no redundant parameters were left.

\subsubsection{Feature relevance analysis and ANN structure optimisation}

Feature relevance is usually assessed by some measure of correlation between the feature and the target variable. The analysis of relevance is complicated by the fact that analysing one feature on its own, as done in univariate feature selection approaches [26], may lead to the removal of elements that are not significantly correlated with the target variable, but that might become highly informative in combination with other features. For this reason, a multivariate method based on the evolutionary ANN Evolver (ANNE) algorithm was used. ANNE is specially designed for the optimisation of ANN classifiers [27, 28] and can be regarded as an embedded feature selection method that simultaneously performs feature selection, ANN structure optimisation and weight training [29].

ANN optimisation and feature selection were carried out for the processing disturbances classification task, and then, the results were re-used for the wettability prediction task. A Multi-Layer Perceptron (MLP) ANN [30] was used as classifier in the first task, and predictor in the second. Preliminary tests revealed that one hidden layer of units was enough to attain a very high accuracy.

ANNE was run using the group of surface parameters obtained after redundancy analysis and thus was employed only for relevance-based feature selection. The feature relevance selection and ANN optimisation procedure consisted of two stages as shown in Fig. 3. In the first stage, ANNE was used to optimise the MLP structure, that is, to define the size of its hidden layer 
Fig. 3 Steps of feature relevance analysis split into two stages. In the first stage (blue lines), the ANNE procedure was used to optimise the MLP structure and generate candidate groups of surface parameters. In the second stage (red lines), the parameter groups were evaluated on the learning results of MLP (using BP training) and a final minimal group of relevant areal surface parameters is generated

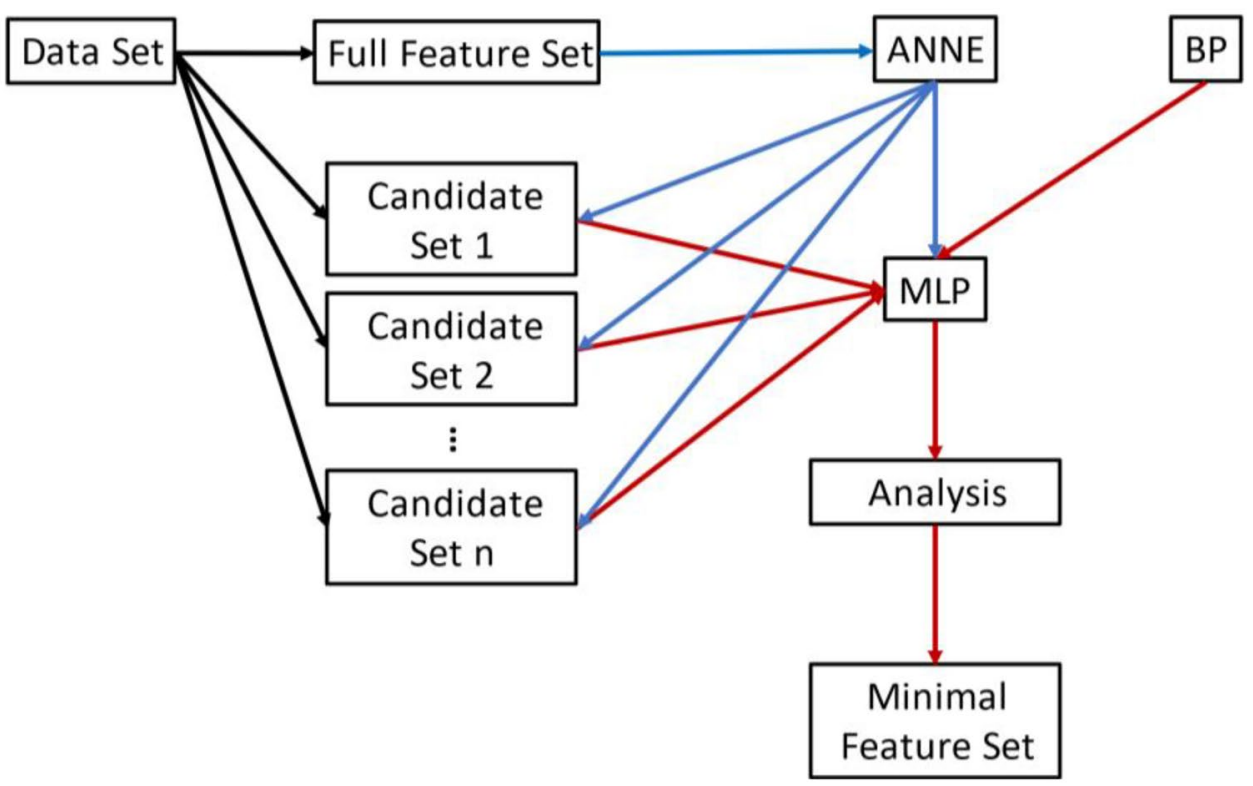

and to evolve minimal sets of relevant areal parameters. In the second stage, the MLP structure was set to the optimal configuration evolved by ANNE. Exploiting the feature selection results from ANNE, a number of candidate groups of surface parameters were formed, and their suitability was evaluated on the MLP ability to learn the classification task. The MLP was trained using the standard back-propagation (BP) procedure [31]. The main parameters of the MLP, and the learning parameters of the ANNE and BP algorithms were experimentally optimised and are listed in Table 5. The remaining parameters were set as in [27].

A final tuning step was performed to adjust the number of iterations required for the BP procedure because of the different nature of the final learning task. The learning curves were analysed, and the duration of the learning procedure was set in order to avoid overfitting. Training data overfitting occurred in both classification and regression tasks. Hence, the learning procedure had to be restricted to, respectively, 100 and 200 iterations, as stated in Table 5.

Following a common practice, a pre-processing step was performed where the areal surface roughness data were normalised using the mean-variance procedure. Due to the stochastic variability of the learning procedure, 10 independent runs of the ANNE algorithm were performed for each experiment, and the results were statistically analysed. For each learning trial, the data set (Set A, Set GAN, or Set A + GAN) was randomly divided into a training set containing $80 \%$ of the samples, and a validation set containing the remaining $20 \%$. For the BP algorithm, 100 independent runs of the procedure were performed for each experiment. The reason for the different number of repetitions is the computational cost associated with the two algorithms, in detail about $16 \mathrm{~min}$ for ANNE and $3 \mathrm{~s}$ for the BP algorithm.

Table 5 MLP architecture and parameterisation of the ANNE and BP algorithms

\begin{tabular}{ll}
\hline MLP & \\
\hline Input nodes & $*$ \\
Hidden nodes & $*$ \\
Output nodes & 3 \\
Transfer function hidden nodes & hypertangent \\
Transfer function output nodes & sigmoidal \\
ANNE & \\
Population size & 200 \\
Iterations & 5,000 \\
Crossover rate on binary mask & 1 \\
Crossover rate on real-valued string & No crossover \\
Mutation rate on binary mask & 0.1 \\
Mutation rate on real-valued string & 0.1 \\
BP rate (problem-specific operator) & 1 \\
Cycles of BP learning per iteration & 1 \\
Selection scheme & Adaptive [32] \\
BP RULE & \\
Iterations (Feature Selection) & 3000 \\
Iterations (Classification Task 1) & 100 \\
Iterations (Regression Task 2) & 200 \\
Learning rate & 0.01 \\
Momentum term & 0.1 \\
$*$ Evolved by ANNE & \\
\hline
\end{tabular}




\section{Results and discussion}

\subsection{Datasets}

Examples of LIPSS topographies from Set A and artificially generated topographies from Set GAN with and without the presence of processing disturbances with the respective average CA values are presented in Fig. 4. The influence of FOD and BIA on the LIPSS characteristics is clearly visible. The FOD increase entailed a decrease in LIPSS amplitudes that eventually led to spots where LIPSS were no longer generated, e.g. when $\mathrm{FOD}=0.8 \mathrm{~mm}$. In regards to the influence of BIA, two types of ripple periodicities were present on the surface, which is typical for LIPSS generated with a p-type polarised beam that is not normal to the surface [33]. Samples produced with lower BIA resulted in a dominant periodicity above the one achieved with optimised laser settings, while higher BIAs led to only smaller periods. Generally, the LIPSS topographies selected for the representative Set A, and consequently the ones generated by the GAN, had widely varied dimensional characteristics, which led to diverse areal surface parameters values and altered their wettability. All of the laser-treated surfaces showed hydrophilic behaviour and the obtained CA values ranged from 26 to
$80 \mathrm{deg}$, with a measurement uncertainty of approximately $10 \mathrm{deg}$. In Table 6, the number of input topographies in each Set, the distributions of the classes and the range of output CA values are summarised.

\subsection{Feature redundancy analysis}

The correlation analysis revealed that several features, i.e. aerial surface roughness parameters, were redundant in all Sets, i.e. Set A, Set GAN and the largest Set B. The analysis of Set B was done only for reference purposes and kept for validation only. The redundancy analysis performed on the small Set A differs from that conducted on Set B. Out of 210 pairwise feature redundancy checks, $23(11 \%)$ were different. Overall, despite some discrepancies, the analysis performed on Set A was in good agreement with the one performed on Set B. Thus, it can be judged that Set A is a representative example of the larger population of Set B. The analysis performed on Set GAN also differed from the distribution of Set B. It should be noted that Set GAN was created using the samples of Set A, and thus 'inherited' the inaccuracies of the latter. Out of 210 pairwise feature redundancy results, the analyses on Set A and Set GAN differed in 30 cases (14\%). The results show a satisfactory agreement between the two sets, indicating that the GAN technique of
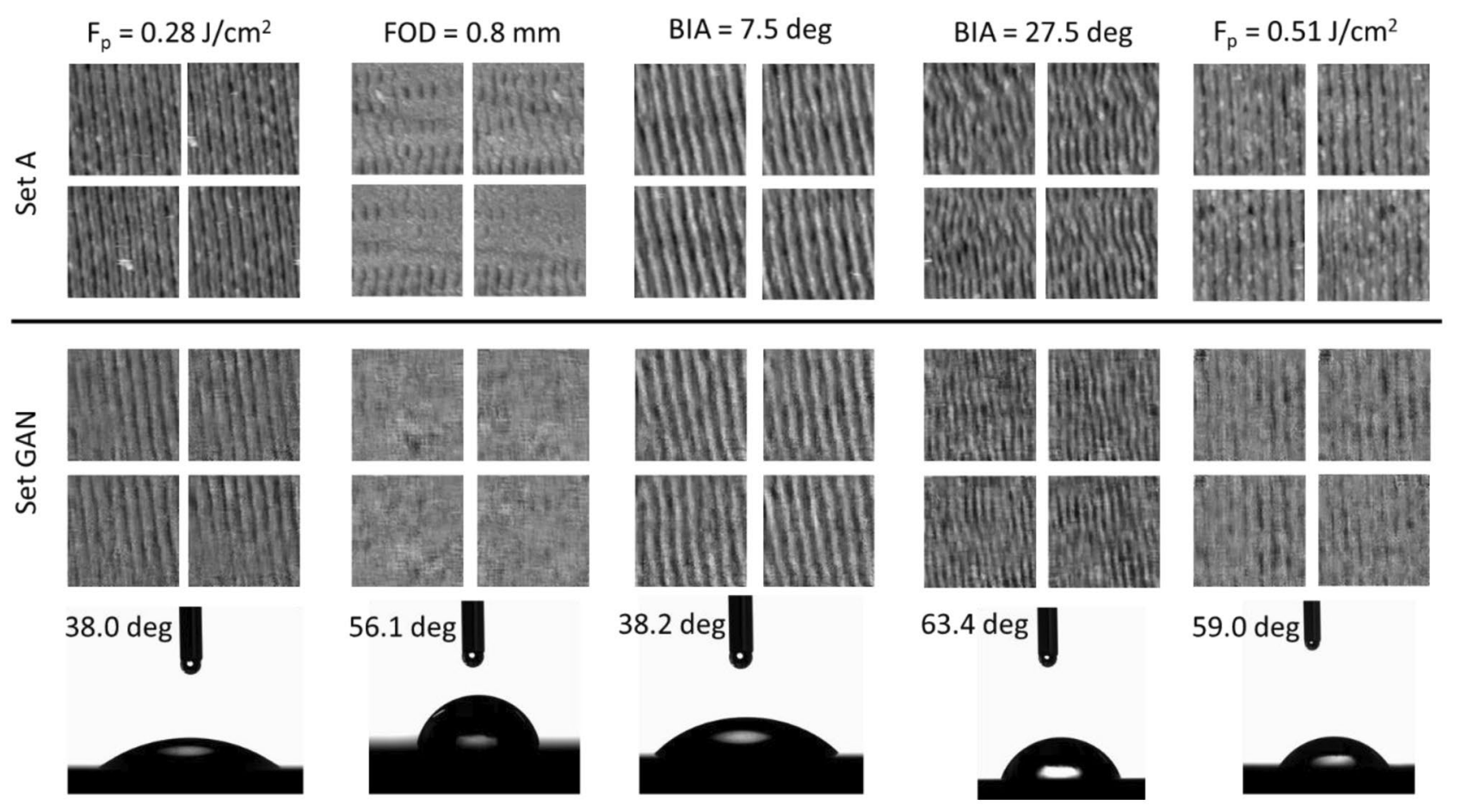

Fig. 4 Examples of $7.8 \mu \mathrm{m} \times 7.8 \mu \mathrm{m}$ LIPSS topographies with and without processing disturbances from Set A together with the respective artificially generated ones from Set GAN. Peak fluence of
$\mathrm{Fp}=0.28 \mathrm{~J} / \mathrm{cm} 2$ was used to produce the surfaces with varying FOD and BIA. Below the images of the water droplets on the laser structured surface with their respective average CA values are presented 
Table 6 Summary of LIPSS topographies within Sets A, GAN and B that was used to classify laser processing disturbances (the first task). Class $\mathrm{N}$ refers to samples produced without processing disturbances but with varying peak fluence. The output values of minimum and maximum CAs are also provided for the regression task, i.e. the wettability prediction

\begin{tabular}{lccc}
\hline & Set A & Set GAN & Set B \\
\hline Total topographies & 288 & 360 & 1104 \\
ISO parameters & 21 & & \\
Task 1: Processing & Disturbance & & \\
Class FOD & 80 & 100 & \\
Class BIA & 112 & 140 & 384 \\
Class N & 96 & 120 & 560 \\
Task 2: Wettability & Prediction classes) & 160 \\
Min CA, deg & 30.2 & 29.5 & \\
Max CA, deg & 81.1 & 80.6 & 25.7 \\
\hline
\end{tabular}

generating artificial topographies captured reasonably well the statistics of Set A.

Table 7 shows the results of the elimination procedure for the three data sets. Redundancy elimination gave the same results for Set A and Set A+ GAN, leading to a reduction in their attributes, i.e. ISO parameters, from 21 to only 10 . On Set GAN, redundancy elimination reduced the set attributes to 9 , where 6 of them are shared with the other two sets.

\subsection{Feature relevance analysis based on ANNE algorithm}

The averages of the feature selection and structure optimisation results, and the classification accuracies obtained for the validation set (20\% of examples of the data set in consideration) are reported in Table 8. For reference, the results obtained using the full set of the ISO parameters are also included in the table. The frequency of each data attribute that was selected in the 10 runs of ANNE is shown in Table 9 for the three data sets.

The results, presented in Table 8, obtained using the three data sets indicated that some ISO parameters might be further discarded due to being less relevant. However, it is important to note that the actual ISO parameters selected differed from set to set. Though, there was a considerable agreement in the size of the surface parameters group using the full 21 ISO parameters and the reduced group after redundancy analysis. In general, when all surface parameters were considered, ANNE tended to select slightly more relevant attributes. In terms of the selected ISO parameters and their selection frequency, the results obtained considering all or a reduced group of parameters, as shown in Table 9, cannot be compared. This is due to the fact that redundant attributes are equally likely to be selected, and the selected frequency is not necessarily an indication of their relevance.

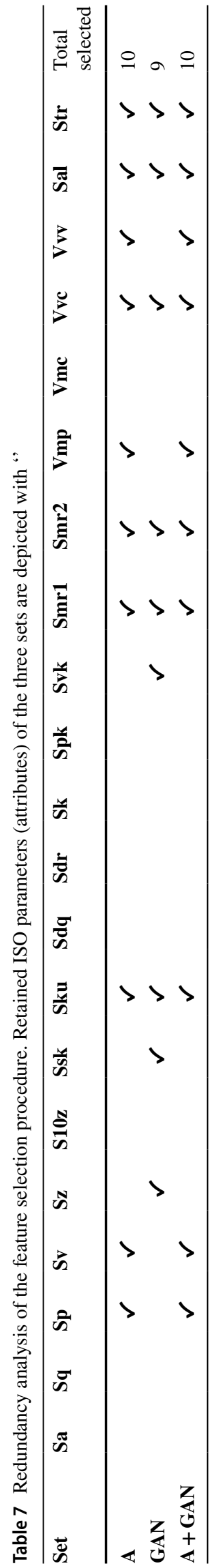


Table 8 Feature selection and structure optimisation results (ANN hidden nodes) obtained by the ANNE algorithm for the three sets. A summary of the classification accuracies achieved for Task 1 on the validation set ( $20 \%$ of examples of the data set in consideration) is included in the table, too. The results are calculated over 10 runs of the algorithm. In the table, 'all' refers to the trials run using the full 21 surface parameters, 'reduced' refers to the parameters group obtained after the redundancy analysis. The significance of the differences in the classification accuracies obtained using the full and reduced ISO parameters is analysed using Mann-Whitney tests and the $\mathrm{p}$-values are provided in the table

\begin{tabular}{|c|c|c|c|c|c|c|}
\hline & \multicolumn{2}{|l|}{ Set A } & \multicolumn{2}{|c|}{ Set GAN } & \multicolumn{2}{|c|}{ Set A + GAN } \\
\hline & all & reduced & all & reduced & all & reduced \\
\hline Selected Features & 6.20 & 5.30 & 6.90 & 5.40 & 9.00 & 8.10 \\
\hline Hidden nodes & 3.40 & 2.9 & 3.10 & 2.70 & 4.00 & 4.50 \\
\hline Min & 79.31 & 46.55 & 86.11 & 84.72 & 87.60 & 89.15 \\
\hline Q1 & 83.19 & 50.86 & 90.63 & 93.06 & 89.34 & 90.12 \\
\hline Median & 85.34 & 55.17 & 93.06 & 94.44 & 94.57 & 91.86 \\
\hline Q3 & 86.21 & 58.62 & 95.83 & 95.83 & 96.51 & 93.02 \\
\hline Max & 89.66 & 68.97 & 98.61 & 100.00 & 97.67 & 98.45 \\
\hline$p-v a l u e$ & $1.7 \times 10^{-4}$ & & 0.73 & & 0.65 & \\
\hline
\end{tabular}

Regarding the classification accuracy, the most evident result is the poor accuracies on Set A attained by ANNE. The analysis of the learning curves did not indicate significant overfitting of the training data. The most plausible explanation is the small size of Set A, which affected ANNE's ability to evolve to high performing solutions. For

Table 9 Selection frequency of each areal surface roughness parameter in the 10 runs of the ANNE algorithm obtained for Sets A, GAN and $\mathrm{A}+\mathrm{GAN}$

\begin{tabular}{llll}
\hline Parameter & Set A & Set GAN & Set A+GAN \\
\hline Sa & 0 & 0 & 0 \\
Sq & 0 & 0 & 0 \\
Sp & 0.1 & 0 & 0.6 \\
Sv & 0.3 & 0 & 1 \\
Sz & 0 & 0.3 & 0 \\
S10z & 0 & 0 & 0 \\
Ssk & 0 & 0.2 & 0 \\
Sku & 0.9 & 0.6 & 0.8 \\
Sdq & 0 & 0 & 0 \\
Sdr & 0 & 0 & 0 \\
Sk & 0 & 0 & 0 \\
Spk & 0 & 0 & 0 \\
Svk & 0 & 1 & 0 \\
Smr1 & 0.3 & 1 & 1 \\
Smr2 & 0.4 & 0.5 & 0.8 \\
Vmp & 0.5 & 0 & 0.5 \\
Vmc & 0 & 0 & 0 \\
Vvc & 0.2 & 0.7 & 0.7 \\
Vvv & 1 & 0 & 0.7 \\
Sal & 0.7 & 0.1 & 1 \\
Str & 0.9 & 1 & 1 \\
Total Selected & 5.3 & 5.40 & \\
\hline & & & 0.10 \\
\hline
\end{tabular}

Sets GAN and A + GAN, the results suggest that MLP could be trained to identify the processing disturbances with high accuracy. There was no distinguishable difference in the accuracy between the results obtained using all or only a smaller subset of attributes, i.e. ISO parameters.

\subsection{Evaluation of candidate surface parameters subsets}

Based on the results obtained using ANNE, the MLP structure was fixed to one hidden layer of 5 nodes even though it was slightly larger than proposed in Table 8 . The reason for this was that the smaller Set A alone might under-represent the complexity of LIPSS topographies.

Using the results in Table 9, a number of candidate ISO parameter groups were created for each of the three sets as shown in Table 10. These candidate groups were based on the selection frequencies, starting with a minimal subset of most frequently selected ISO parameters and successively adding more attributes. These candidate sets were evaluated on the learning results of the MLP after it was trained using BP and with only the selected ISO parameters. The results are shown for each ISO parameters' group and data set in Table 11. For the sake of comparison, the results include those obtained using the full set and the set generated after redundancy analysis.

In terms of classification accuracy, the results reported in Table 11 are in good agreement with those obtained using ANNE and confirm again that high accuracy results can be obtained with a significantly reduced number of surface parameters. The classification accuracies obtained using Set A are lower than those obtained using the other two data sets, although the differences are significantly smaller than those recorded for ANNE. 
Table 10 Candidate surface parameters groups tested on data Sets A, GAN and A+GAN. Their size is indicated by their group coding in the first column (e.g. $F_{6}$ has six ISO parameters). Selected parameters in the group are indicated by "'

\begin{tabular}{|c|c|c|c|c|c|c|c|c|c|c|c|c|c|c|c|c|c|c|c|c|}
\hline Group Sa & Sq & Sp & $\mathbf{S v}$ & $\mathbf{S z}$ & S10z & Ssk & Sku & Sdq & Sdr & Sk & Spk & Svk & Smr1 & Smr2 & Vmp & Vmc & Vve & Vvv & Sal & Str \\
\hline \multicolumn{21}{|l|}{ Set A } \\
\hline $\boldsymbol{F}_{3}$ & & & & & & & $\checkmark$ & & & & & & & & & & & $\checkmark$ & & $\checkmark$ \\
\hline $\boldsymbol{F}_{4}$ & & & & & & & $\checkmark$ & & & & & & & & & & & $\checkmark$ & $\checkmark$ & $\checkmark$ \\
\hline $\boldsymbol{F}_{5}$ & & & & & & & $\checkmark$ & & & & & & & & $\checkmark$ & & & $\checkmark$ & $\checkmark$ & $\checkmark$ \\
\hline $\boldsymbol{F}_{6}$ & & & & & & & $\checkmark$ & & & & & & & $\checkmark$ & $\checkmark$ & & & $\checkmark$ & $\checkmark$ & $\checkmark$ \\
\hline $\boldsymbol{F}_{10}$ & & $\checkmark$ & $\checkmark$ & & & & $\checkmark$ & & & & & & $\checkmark$ & $\checkmark$ & $\checkmark$ & & $\checkmark$ & $\checkmark$ & $\checkmark$ & $\checkmark$ \\
\hline \multicolumn{21}{|l|}{ Set GAN } \\
\hline $\boldsymbol{F}_{3}$ & & & & & & & & & & & & $\checkmark$ & $\checkmark$ & & & & & & & $\checkmark$ \\
\hline $\boldsymbol{F}_{4}$ & & & & & & & & & & & & $\checkmark$ & $\checkmark$ & & & & $\checkmark$ & & & $\checkmark$ \\
\hline $\boldsymbol{F}_{5}$ & & & & & & & $\checkmark$ & & & & & $\checkmark$ & $\checkmark$ & & & & $\checkmark$ & & & $\checkmark$ \\
\hline $\boldsymbol{F}_{6}$ & & & & & & & $\checkmark$ & & & & & $\checkmark$ & $\checkmark$ & $\checkmark$ & & & $\checkmark$ & & & $\checkmark$ \\
\hline $\boldsymbol{F}_{9}$ & & & & $\checkmark$ & & $\checkmark$ & $\checkmark$ & & & & & $\checkmark$ & $\checkmark$ & $\checkmark$ & & & $\checkmark$ & & & $\checkmark$ \\
\hline \multicolumn{21}{|c|}{ Set A + GAN } \\
\hline $\boldsymbol{F}_{4}$ & & & $\checkmark$ & & & & & & & & & & $\checkmark$ & & & & & & $\checkmark$ & $\checkmark$ \\
\hline $\boldsymbol{F}_{6}$ & & & $\checkmark$ & & & & $\checkmark$ & & & & & & $\checkmark$ & $\checkmark$ & & & & & $\checkmark$ & $\checkmark$ \\
\hline $\boldsymbol{F}_{8}$ & & & $\checkmark$ & & & & $\checkmark$ & & & & & & $\checkmark$ & $\checkmark$ & & & $\checkmark$ & $\checkmark$ & $\checkmark$ & $\checkmark$ \\
\hline $\boldsymbol{F}_{9}$ & & $\checkmark$ & $\checkmark$ & & & & $\checkmark$ & & & & & & $\checkmark$ & $\checkmark$ & & & $\checkmark$ & $\checkmark$ & $\checkmark$ & $\checkmark$ \\
\hline $\boldsymbol{F}_{10}$ & & $\checkmark$ & $\checkmark$ & & & & $\checkmark$ & & & & & & $\checkmark$ & $\checkmark$ & $\checkmark$ & & $\checkmark$ & $\checkmark$ & $\checkmark$ & $\checkmark$ \\
\hline
\end{tabular}

Table 11 shows that the removal of redundant features had marginal to no effect on the learning accuracy of the classifier for the data sets that included the artificial topographies.
On Set A, the differences are more marked although still moderate. The same effect was observed after the elimination of irrelevant ISO parameters on the learning results of
Table 11 A summary of the MLP classification accuracies obtained on the validation set ( $20 \%$ of the whole data set) using the parameter groups in Table 10. For each data set, the statistics refer to 100 learning trials using the $\mathrm{BP}$ algorithm. For reference, also the results of training the MLP using all ISO surface parameters are given. The significance of the differences in the classification accuracies obtained using the all and candidate attribute sets is analysed using pairwise MannWhitney tests and reported by the p-values

\begin{tabular}{|c|c|c|c|c|c|c|}
\hline \multicolumn{7}{|l|}{ Set A } \\
\hline & $F_{3}$ & $F_{4}$ & $F_{5}$ & $F_{6}$ & $F_{10}$ & $F_{\text {All }}$ \\
\hline Min & 70.69 & 70.69 & 70.69 & 72.41 & 77.59 & 81.03 \\
\hline Q1 & 77.59 & 77.59 & 79.31 & 84.48 & 87.93 & 91.38 \\
\hline Median & 81.03 & 81.03 & 82.76 & 86.21 & 89.66 & 93.10 \\
\hline Q3 & 83.19 & 84.48 & 86.21 & 89.66 & 93.10 & 94.83 \\
\hline $\operatorname{Max}$ & 89.66 & 93.10 & 91.38 & 96.55 & 96.55 & 100.00 \\
\hline p-value & 0 & 0 & 0 & 0 & 0 & \\
\hline \multicolumn{7}{|c|}{ Set GAN } \\
\hline & $F_{3}$ & $F_{4}$ & $F_{5}$ & $F_{6}$ & $F_{9}$ & $F_{\text {All }}$ \\
\hline Min & 75.00 & 84.72 & 84.72 & 83.33 & 83.33 & 84.72 \\
\hline Q1 & 83.33 & 90.28 & 91.67 & 93.06 & 94.44 & 95.49 \\
\hline Median & 86.11 & 94.44 & 95.83 & 95.83 & 96.53 & 97.22 \\
\hline Q3 & 88.89 & 95.83 & 97.22 & 97.22 & 97.22 & 98.61 \\
\hline Max & 94.44 & 100.00 & 100.00 & 100.00 & 100.00 & 100.00 \\
\hline$p$-value & 0 & 0 & 0.0065 & 0.0767 & 0.1721 & \\
\hline \multicolumn{7}{|c|}{ Set A + GAN } \\
\hline & $F_{4}$ & $F_{6}$ & $F_{8}$ & $F_{9}$ & $F_{10}$ & $F_{A l l}$ \\
\hline Min & 70.54 & 78.29 & 83.72 & 86.05 & 84.50 & 82.95 \\
\hline Q1 & 75.97 & 88.37 & 89.92 & 89.92 & 91.47 & 92.05 \\
\hline Median & 79.07 & 90.70 & 91.47 & 92.25 & 93.02 & 93.80 \\
\hline Q3 & 82.17 & 93.02 & 93.80 & 93.80 & 94.57 & 96.12 \\
\hline Max & 88.37 & 96.90 & 98.45 & 97.67 & 99.22 & 99.22 \\
\hline$p$-value & 0 & 0 & 0 & 0 & 0.0338 & \\
\hline
\end{tabular}


the classifier. The resulting classification accuracies were most sensitive for removing irrelevant surface parameters for Set A. This was likely due to the small size of the data set, which made accurate MLP learning and evaluation more difficult.

The results in Table 11 suggest that the feature selection affects the classifier performance mostly for Set A. The most conservative choice would be to use the group of nonredundant ISO parameters $F_{10}$, or if some further reduction in performance is acceptable, the parameter group $F_{6}$ could be adopted. If Set GAN is used, the surface parameters can be trimmed down to the six data attributes of $F_{6}$ without significantly affecting the performance. If Set A+GAN is utilised, the tests show that the classifier accuracy will suffer only a very modest deterioration (less than 1\%) using group $F_{10}$ of non-redundant ISO parameters and only modest (around $2 \%$ ) using $F_{8}$ data attributes. These final choices are validated in the last step, where the MLP is tested against the previously unseen Set B.

\subsection{Task 1: Classification of laser processing disturbances}

Figure 5 reports the results on accuracies, achieved in identifying the processing disturbances, of the classifiers obtained after 100 independent runs of the BP algorithm on Sets A, GAN, and A + GAN while the validation was performed on Set B. In general, the accuracy results were inferior to those obtained in the feature selection steps (as shown in Table 11). The deterioration of the performance was most dramatic in the learning trials performed using only Set GAN, and least severe when only Set A was used. Set A+ GAN achieved only slightly worse accuracy compared to Set A. It is worth stating that the learning

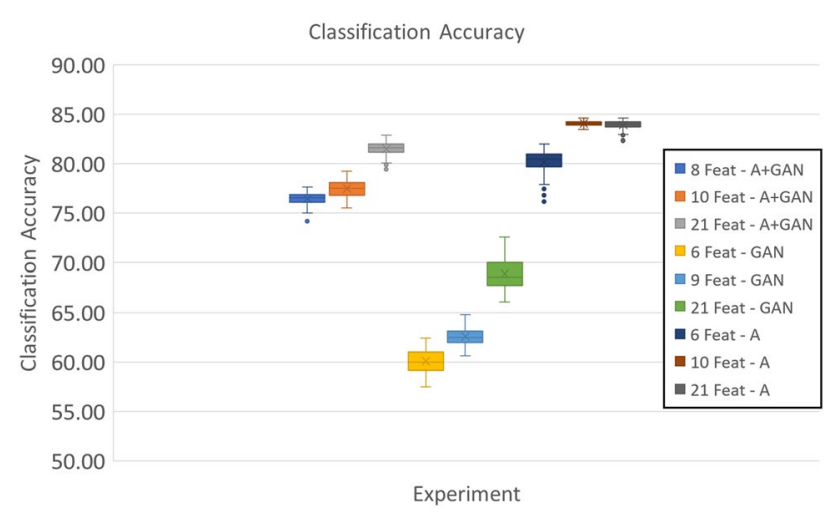

Fig. 5 Results achieved on classification of laser processing disturbances. Accuracies obtained in three experiments where feature selection and BP training were performed respectively on Sets A, GAN, and A+GAN. The MLP learning procedure was validated on previously unseen Set B. Three surface parameters groups were tested per each data set tasks in the feature selection and classification stages were different, i.e. the first requiring generalisation to unseen samples of already introduced surfaces, and the second generalisation to different samples of previously unseen surfaces. The lower classification accuracies achieved in the latter experiments are likely to reflect the more challenging nature of the task.

The artificial LIPSS topographies generated applying GAN appeared to capture at least partly the overall characteristics of Set A. However, MLPs trained on Set GAN were very poor at generalising the learning results when applied on Set B. This result shows that the GAN-generated data were not representative of the full distribution of Set B. Given that MLPs trained using Set A did generalise well, the results seem to indicate that the problem lies within the GAN procedure itself, rather than the poor quality of the scans that were fed to GAN. One reason for this result may be that the GAN learning process had been interrupted too early. At present, the duration of the GAN learning reflected a trade-off between the computational cost and the visual appearance. Further tests could investigate whether it is worth the GAN learning time to be extended.

Since the use of data samples from real surface scans produced the best learning results, the next step was to use Set A to re-train the MLP using the ISO parameters groups selected using Set GAN and Set A+ GAN. This experiment aimed at evaluating the goodness of the feature selection results obtained using artificial data samples. The results, shown in Fig. 6, are very similar, with average classification accuracies mostly ranging between 82 and $84 \%$. The only exception was the learning trials performed using the minimal parameter group $F_{6}$ that was selected using Set A, where the average accuracy was $80.4 \%$.

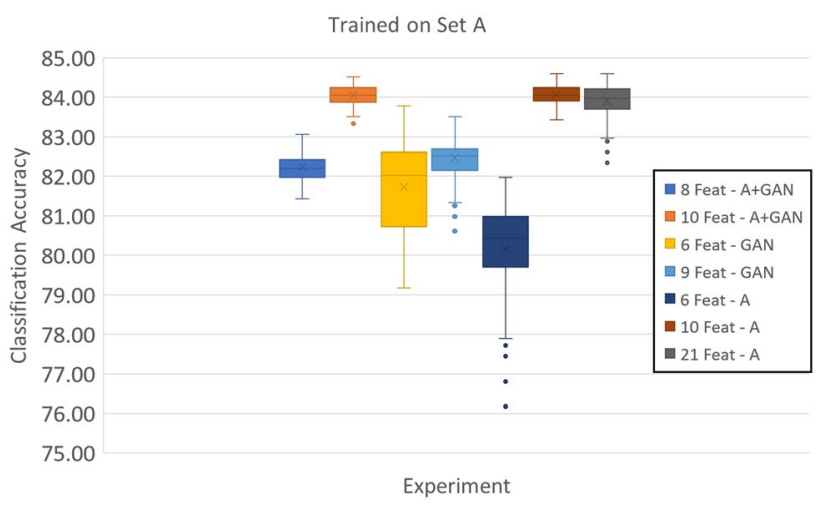

Fig. 6 Results of the classification task with MLPs being trained only on Set A using the parameter groups identified for Sets GAN and $\mathrm{A}+\mathrm{GAN}$ and the validation performed on previously unseen Set B. Two surface parameters groups were tested per each dataset and the results compared to those obtained using all 21 surface parameters Sets $\mathrm{A}$ and $\mathrm{A}+\mathrm{GAN}$ had the same parameter group $F_{10}$, as indicated in Table 10 
Table 12 Confusion matrices of processing disturbances classification results presented in Fig. 6. The MLP classifier was trained only on Set A using the surface parameter group with minimal number of attributes selected, respectively, for Sets A, GAN, and A+GAN while the validation was performed on Set B

\begin{tabular}{|c|c|c|c|c|}
\hline \multicolumn{5}{|c|}{ a) Parameters selected using Set A } \\
\hline \multirow[t]{2}{*}{$\boldsymbol{F}_{6}$} & & \multicolumn{3}{|c|}{ Classified as } \\
\hline & & FOD & BIA & $\mathrm{N}$ \\
\hline \multirow[t]{3}{*}{ class } & FOD & 281.68 & 75.5 & 26.82 \\
\hline & BIA & 47.65 & 505.9 & 6.45 \\
\hline & $\mathrm{N}$ & 21.2 & 41.34 & 97.46 \\
\hline \multicolumn{5}{|c|}{ b) Parameters selected using Set GAN } \\
\hline \multirow[t]{2}{*}{$\boldsymbol{F}_{6}$} & & \multicolumn{3}{|c|}{ Classified as } \\
\hline & & FOD & BIA & $\mathrm{N}$ \\
\hline \multirow[t]{3}{*}{ class } & FOD & 260.96 & 95.7 & 27.34 \\
\hline & BIA & 13.85 & 540.03 & 6.12 \\
\hline & $\mathrm{N}$ & 30.73 & 27.98 & 101.29 \\
\hline \multicolumn{5}{|c|}{ c) Parameters selected using Set A+GAN } \\
\hline \multirow[t]{2}{*}{$\boldsymbol{F}_{8}$} & & \multicolumn{3}{|c|}{ Classified as } \\
\hline & & FOD & BIA & $\mathrm{N}$ \\
\hline \multirow[t]{3}{*}{ class } & FOD & 278.33 & 80.64 & 25.03 \\
\hline & BIA & 22.5 & 531.79 & 5.71 \\
\hline & $\mathrm{N}$ & 22.94 & 39.39 & 97.67 \\
\hline
\end{tabular}

The best learning results were obtained using surface parameter group $F_{10}$, i.e. the non-redundant data attributes selected after analysing Sets A and A+GAN. The removal of irrelevant surface parameters had instead a statistically significant negative effect on the accuracy results, although in practical terms this was very modest.

Table 12 reports the confusion matrices for the classification results on Set B presented in Fig. 6, using MLPs trained on Set A by using the minimal parameter groups $F_{6}$ (selected on Set A), $F_{6}$ (selected on Set GAN), and $F_{8}$ (selected on Set A+GAN). The largest source of misclassifications was due to FOD topographies being identified as BIA. In proportion to the number of examples per class, the largest sources of incorrect classifications were samples from class $\mathrm{N}$ identified as BIA in the case of the surface parameters $F_{6}$ of Sets A and A + GAN, and FOD identified as BIA for $F_{8}$ obtained using Set GAN. It was also observed that for three tested cases a similar number of samples from class FOD were identified as class $\mathrm{N}$. This could be attributed to the supplementary LIPSS samples produced with laser peak fluence close to the ripples' threshold, which had similar topographies to the ones obtained with higher FOD. Hence, those samples were more prone to be misclassified.

\subsection{Task 2: Contact angle prediction}

Figure 7 shows the root mean square (r.m.s.) values of the CA predictions from the validation step performed on Set B. Similar to the processing disturbance classification task, a further experiment was run employing Set A to train MLP by using the ISO parameter groups selected

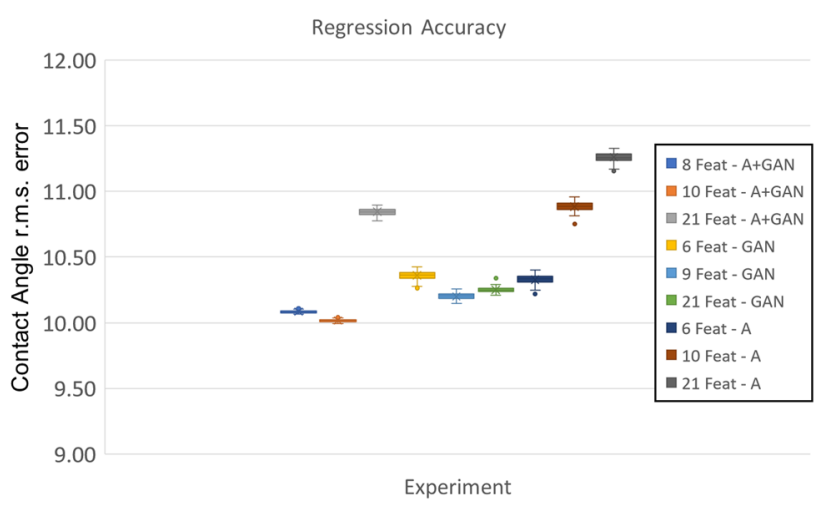

Fig. 7 Root mean square (r.m.s.) accuracy results for the CA prediction task from three experiments where feature selection and BP training were performed respectively on data sets A, GAN, and A+GAN, while the learning results were validated on previously unseen Set B 


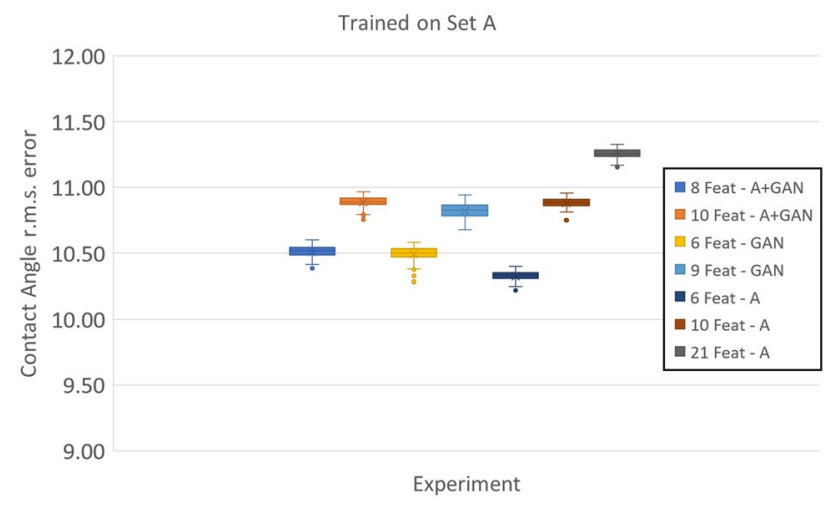

Fig. 8 Accuracy results for the CA prediction task with MLPs being trained on Set A using the parameter groups identified for Sets GAN and A + GAN and validated on Set B. Two surface parameters groups were tested per each dataset and the results compared to those obtained using all 21 surface parameters

using Sets GAN and Set A + GAN. The results of this last experiment are given in Fig. 8.

The results presented in Fig. 8 are fairly similar with the average (r.m.s.) error of around 11 degrees for all combinations of training data sets and ISO parameter groups. In general, feature selection helped the MLPs to learn the CA prediction with marginally better results. Data augmentation appeared also to play a beneficial role, since the best accuracy results were obtained by training the MLPs using Set A+GAN. Although statistically significant, it should be noted that the measured differences in accuracy were always within 1.5 degrees. The best results were obtained when training the MLPs on the augmented Set A + GAN of examples and using ISO parameter groups $F_{8}$ or $F_{10}$ to describe the samples. In general, it can be stated that the average r.m.s. values obtained in the experiments are comparable with the CA measurement uncertainty, and most probably this limited the MLP learning abilities. Obtaining a more accurate CA measurements might improve the MLP training and allow the MLP to differentiate better the usefulness of different data sets and ISO parameter groups.

\section{Conclusions}

In this research, an approach is presented for applying ANNs to classification and prediction tasks when ultrafast laser surface structuring/texturing is performed. ANN tools were developed and validated for pre- and post-processing of laser surface treatment data, especially areal surface roughness parameters of LIPSS topographies, that proved to be sufficiently effective. In particular, high prediction accuracies were achieved by MLP classifiers on the detection of laser processing disturbances that affect the LIPSS generation. MLPs were also used to predict with high accuracy the functional response, i.e. wettability, of LIPSS-treated surfaces.
Regarding the applied ANN tools, using a small experimental dataset augmented with GAN-created artificial topographies proved to be beneficial for the tool's development. GAN-generated data were especially valuable when utilised for feature relevance analysis employing the evolutionary ANNE algorithm. Even if GAN-based artificial data reproduced well the statistics of real samples, the GAN-generated topographies were less useful in supporting the MLP generalisation capabilities on the laser processing disturbances classification task. That was attributed to the GAN insufficient learning process, especially its premature interruption.

A range of feature selection methods were applied. By combining their capabilities, it was possible to identify the number of salient aerial roughness parameters needed to characterise the surfaces, without any significant negative effect on the MLP performance. Specifically, feature redundancy analysis revealed that the initial 21 ISO parameters can be narrowed down to only 10 , and such a small subset of data attributes was enough to achieve a high MLP prediction accuracy, especially in the laser processing disturbances classification task. Further trimming of irrelevant attributes down to an even smaller subsets of 6 or 8 surface parameters led to fairly similar prediction accuracies. Such substantial scale downs of data attributes can have a valuable impact on the practical aspects of data acquisition procedures, because it can reduce the number of costly, time-consuming, and sometimes complex measurements.

Finally, the ANN validation part on a larger unseen dataset showed that identification of processing disturbances could be accomplished with accuracy close to $85 \%$. The wettability of LIPSS-treated surfaces was predicted within the static water CA measurement uncertainty of approximately 10 degrees. Considering those encouraging findings, it can be concluded that the developed ANN-based tools can represent a generic approach for monitoring the LIPSS treatment operations. These tools can map the resulting areal parameters of processed surfaces to any disturbances present during the process, and consequently also to their desired functional performance.

Supplementary information The online version contains supplementary material available at https://doi.org/10.1007/s00170-021-08589-9.

Author contribution Luca Baronti involved in conceptualisation, methodology, software, validation, formal analysis, investigation, data curation, writing — original draft; Aleksandra Michalek took part in conceptualisation, methodology, investigation, validation, writing — original draft, visualisation.; Marco Castellani involved in conceptualisation, methodology, software, validation, formal analysis, investigation, writing —original draft, visualisation, supervision; Pavel Penchev took part in conceptualisation, methodology, experimental trials, data collection; Tian Long See took part in supervision; Stefan Dimov involved in conceptualisation, methodology, writing—review \& editing, supervision. 
Funding The work was carried out within the H2020 FoF program "High-Impact Injection Moulding Platform for mass-production of 3D and/or large micro-structured surfaces with Antimicrobial, Selfcleaning, Anti-scratch, Anti-squeak and Aesthetic functionalities" (HIMALAIA) and of the UKIERI DST program "Surface functionalization for food, packaging, and healthcare applications". Also, the authors would like to thank the Manufacturing Technology Center (MTC) for the financial support of Aleksandra Michalek's Ph.D. research.

Data availability Additional data are available in the Supplementary Material.

\section{Declarations}

Ethics approval Not applicable.

Consent to participate Not applicable.

Consent for publication Not applicable.

Competing interests The authors declare no competing interests.

Open Access This article is licensed under a Creative Commons Attribution 4.0 International License, which permits use, sharing, adaptation, distribution and reproduction in any medium or format, as long as you give appropriate credit to the original author(s) and the source, provide a link to the Creative Commons licence, and indicate if changes were made. The images or other third party material in this article are included in the article's Creative Commons licence, unless indicated otherwise in a credit line to the material. If material is not included in the article's Creative Commons licence and your intended use is not permitted by statutory regulation or exceeds the permitted use, you will need to obtain permission directly from the copyright holder. To view a copy of this licence, visit http://creativecommons.org/licenses/by/4.0/.

\section{References}

1. Kumar K, Thakur GSM (2012) Advanced Applications of Neural Networks and Artificial Intelligence: A Review. Int J Inf Technol Comput Sci 4:57-68. https://doi.org/10.5815/ijitcs.2012.06.08

2. Meireles MRG, Almeida PEM, Simões MG (2003) A comprehensive review for industrial applicability of artificial neural networks. IEEE Trans Ind Electron 50:585-601. https://doi.org/10. 1109/TIE.2003.812470

3. Paliwal M, Kumar UA (2009) Neural networks and statistical techniques: A review of applications. Expert Syst Appl 36:2-17. https://doi.org/10.1016/j.eswa.2007.10.005

4. Desai CK, Shaikh A (2012) Prediction of depth of cut for singlepass laser micro-milling process using semi-analytical, ANN and GP approaches. Int J Adv Manuf Technol 60:865-882. https://doi. org/10.1007/s00170-011-3677-8

5. Karazi SM, Issa A, Brabazon D (2009) Comparison of ANN and $\mathrm{DoE}$ for the prediction of laser-machined micro-channel dimensions. Opt Lasers Eng 47:956-964. https://doi.org/10.1016/j.optlaseng. 2009.04.009

6. Campanelli SL, Casalino G, Ludovico AD, Bonserio C (2013) An artificial neural network approach for the control of the laser milling process. Int J Adv Manuf Technol 66:1777-1784. https:// doi.org/10.1007/s00170-012-4457-9

7. Teixidor D, Grzenda M, Bustillo A, Ciurana J (2015) Modeling pulsed laser micromachining of micro geometries using machine-learning techniques. J Intell Manuf 26:801-814. https:// doi.org/10.1007/s10845-013-0835-x

8. Shashank V, Pardha Saradhi V, Jagadesh T (2019) Modeling of Laser assisted machining process using Artificial Neural Network, in: J Phys Conf Ser. https://doi.org/10.1088/1742-6596/1172/1/ 012040

9. Yousef BF, Knopf GK, Bordatchev EV, Nikumb SK (2003) Neural network modeling and analysis of the material removal process during laser machining. Int J Adv Manuf Technol 22:41-53. https://doi.org/10.1007/s00170-002-1441-9

10. Gonzalez-Val C, Pallas A, Panadeiro V, Rodriguez A (2020) A convolutional approach to quality monitoring for laser manufacturing. J Intell Manuf 31:789-795. https://doi.org/10.1007/ s10845-019-01495-8

11. Luo H, Zeng H, Hu L, Hu X, Zhou Z (2005) Application of artificial neural network in laser welding defect diagnosis. J Mater Process Technol 170:403-411. https://doi.org/10.1016/j. jmatprotec.2005.06.008

12. Tani S, Aoyagi Y, Kobayashi Y (2020) Neural-network-assisted in situ processing monitoring by speckle pattern observation. Opt Express 28:26180. https://doi.org/10.1364/oe.400785

13. Feng S, Zhou H, Dong H (2019) Using deep neural network with small dataset to predict material defects. Mater Des 162:300-310. https://doi.org/10.1016/j.matdes.2018.11.060

14. Heath DJ, Grant-Jacob JA, Xie Y, Mackay BS, Baker JAG, Eason RW, Mills B (2018) Machine learning for 3D simulated visualization of laser machining. Opt Express 26:21574. https:// doi.org/10.1364/oe.26.021574

15. Isola P, Zhu JY, Zhou T, Efros AA (2017) Image-to-image translation with conditional adversarial networks, Proc. - 30th IEEE Conf Comput Vis Pattern Recognition, CVPR 2017. 2017:5967-5976. https://doi.org/10.1109/CVPR.2017.632.

16. Mills B, Heath DJ, Grant-Jacob JA, Eason RW (2018) Predictive capabilities for laser machining via a neural network. Opt Express 26:17245. https://doi.org/10.1364/OE.26.017245

17. McDonnell MDT, Arnaldo D, Pelletier E, Grant-Jacob JA, Praeger M, Karnakis D, Eason RW, Mills B (2021) Machine learning for multidimensional optimisation and predictive visualisation of laser machining. J Intell Manuf. https://doi.org/10.1007/s10845-020-01717-4

18. Florian C, Kirner SV, Krüger J, Bonse J (2020) Surface functionalization by laser-induced periodic surface structures. J Laser Appl 32:022063. https://doi.org/10.2351/7.0000103

19. Michalek A, Batal A, Qi S, Penchev P, Bruneel D, See TL, Dimov S (2020) Modelling ultrafast laser structuring/texturing of freeform surfaces. Appl Surf Sci Adv 2:100036. https://doi. org/10.1016/j.apsadv.2020.100036

20. Michalek A, Jwad T, Penchev P, See TL, Dimov S (2020) Inline LIPSS monitoring method employing light diffraction. J Micro Nano Manuf 8. https://doi.org/10.1115/1.4045681

21. Batal A, Michalek A, Garcia-Giron A, Nasrollahi V, Penchev P, Sammons R, Dimov S (2020) Effects of laser processing conditions on wettability and proliferation of Saos-2 cells on CoCrMo alloy surfaces. Adv Opt Technol 9:67-78. https://doi.org/10.1515/ aot-2019-0051

22. Garcia-Giron A, Romano JM, Batal A, Michałek A, Penchev P, Dimov SS (2020) Experimental investigation of processing disturbances in laser surface patterning. Opt Lasers Eng 126. https:// doi.org/10.1016/j.optlaseng.2019.105900

23. Sunil AKSB, Jha KP, Kulwant AM, Vivekanand S, Sinha KS (2018) Surface micro-structuring of type 304 stainless steel by femtosecond pulsed laser : effect on surface wettability and corrosion resistance. Appl Phys A 124:1-9. https://doi.org/10.1007/ s00339-018-2243-8

24. Podor R, Le Goff X, Cordara T, Odorico M, Favrichon J, Claparede L, Szenknect S, Dacheux N, Podor R, Le Goff X, Cordara T, Odorico M, Favrichon J, Dacheux N (2019) 3D-SEM height maps series to 
monitor materials corrosion and dissolution To cite this version : HAL Id : hal-02064121. Mater Charact 150:220-228. https://doi. org/10.1016/j.matchar.2019.02.017

25. Moore D, McGabe G, Akman O (2021) Introduction to the practice statistics, 10th edn. Macmillian, NY

26. Bolón-Canedo V, Sánchez-Maroño N, Alonso-Betanzos A (2016) Feature selection for high-dimensional data. Prog Artif Intell 5:65-75. https://doi.org/10.1007/s13748-015-0080-y

27. Castellani M (2013) Evolutionary generation of neural network classifiers-An empirical comparison. Neurocomputing 99:214229. https://doi.org/10.1016/j.neucom.2012.07.010

28. Castellani M (2006) ANNE - A new algorithm for evolution of artificial neural network classifier systems, 2006 IEEE Congr. Evol Comput CEC 2006:3294-3301. https://doi.org/10.1109/cec. 2006.1688728

29. Blum AL, Langley P (1997) Selection of relevant features and examples in machine. Artif Intell 97:245-271
30. Pham DT, Packianather MS, Afify AA (2007) Artificial Neural Networks, in: Comput Intell, Springer US, Boston, MA, 67-92. https://doi.org/10.1007/0-387-37452-3_3

31. Rumelhart DE, McClelland JL (1986) Parallel distributed processing: Explorations in the microstructure of cognition. The MIT Press. https://doi.org/10.7551/mitpress/5236.001.0001

32. Pham DT, Castellani M (2010) Adaptive selection routine for evolutionary algorithms. Proc Inst Mech Eng Part I J Syst Control Eng 224:623-633. https://doi.org/10.1243/09596518JSCE942

33. Young JF, Preston JS, Van Driel HM, Sipe JE (1983) Laserinduced periodic surface structure. II. Experiments on $\mathrm{Ge}, \mathrm{Si}$, Al, and brass. Phys Rev B 27:1155-1172. https://doi.org/10.1103/ PhysRevB.27.1155

Publisher's Note Springer Nature remains neutral with regard to jurisdictional claims in published maps and institutional affiliations. 